\title{
Pacific
}

Journal of

Mathematics

\section{A MEAN CURVATURE ESTIMATE FOR CYLINDRICALLY BOUNDED SUBMANIFOLDS}

Luis J. Alías AND Marcos Dajczer 


\title{
A MEAN CURVATURE ESTIMATE FOR CYLINDRICALLY BOUNDED SUBMANIFOLDS
}

\author{
Luis J. Alías AND Marcos DAJCZER
}

\begin{abstract}
In an earlier article in coauthorship with G. P. Bessa, we obtained an estimate for the mean curvature of a cylindrically bounded proper submanifold in a product manifold where one factor is a Euclidean space. Here we extend this estimate to a general product ambient space endowed with a warped product structure.
\end{abstract}

Let $\left(L^{\ell}, g_{L}\right)$ and $\left(P^{n}, g_{P}\right)$ be complete Riemannian manifolds of dimension $\ell$ and $n$, respectively, where $L^{\ell}$ is noncompact. Then let $N^{n+\ell}=L^{\ell} \times{ }_{\rho} P^{n}$ be the product manifold $L^{\ell} \times P^{n}$ endowed with the warped product metric $d s^{2}=$ $d g_{L}+\rho^{2} d g_{P}$ for some positive warping function $\rho \in C^{\infty}(L)$.

Let $B_{P}\left(r_{0}\right)$ denote the geodesic ball with radius $r_{0}$ centered at a reference point $o \in P^{n}$. Assume that the radial sectional curvatures in $B_{P}\left(r_{0}\right)$ along the geodesics issuing from $o$ are bounded as $K_{P}^{\mathrm{rad}} \leq b$ for some constant $b \in \mathbb{R}$, and that $0<$ $r_{0}<\min \left\{\operatorname{inj}_{P}(o), \pi / 2 \sqrt{b}\right\}$, where $\operatorname{inj}_{P}(o)$ is the injectivity radius at $o$ and $\pi / 2 \sqrt{b}$ is replaced by $+\infty$ if $b \leq 0$. Then the mean curvature of the geodesic sphere $S_{P}\left(r_{0}\right)=\partial B_{P}\left(r_{0}\right)$ can be estimated from below by the mean curvature of a geodesic sphere of a space form of curvature $b$, that is,

$$
C_{b}(t)= \begin{cases}\sqrt{b} \cot (\sqrt{b} t) & \text { if } b>0, \\ 1 / t & \text { if } b=0, \\ \sqrt{-b} \operatorname{coth}(\sqrt{-b} t) & \text { if } b<0 .\end{cases}
$$

This is a direct consequence of the comparison theorems for the Riemannian distance, since the Hessian (respectively, Laplacian) of the distance function is nothing but the second fundamental form (respectively, mean curvature) of the geodesic spheres. A classical reference on this topic is [Greene and Wu 1979]. We also refer the reader to [Petersen 2006] or [Pigola et al. 2008] for a modern approach to the Hessian and Laplacian comparison theorems.

MSC2010: 53C40, 53C42.

Keywords: Cylindrically bounded submanifolds, Omori-Yau maximum principle, proper immersions. 
By a cylinder in the warped space $N^{n+\ell}$, we mean a closed subset of the form

$$
\mathscr{C}_{r_{0}}=\left\{(x, y) \in N^{n+\ell}: x \in L^{\ell} \text { and } y \in B_{P}\left(r_{0}\right)\right\} .
$$

Since the submanifolds $L^{\ell} \times\left\{p_{0}\right\} \subset N^{n+\ell}$ are totally geodesic, we have

$$
\left|\rho H_{\mathscr{C}_{0}}\right| \geq \frac{n-1}{\ell+n-1} C_{b}\left(r_{0}\right),
$$

where $H_{\mathscr{C}_{r_{0}}}$ is the mean curvature vector field of the hypersurface $L^{\ell} \times S_{p}\left(r_{0}\right)$.

The following theorem extends the result in [Alías et al. 2009], where the cylinders under consideration are contained in product spaces $\mathbb{R}^{\ell} \times P^{n}$. After the statement, we recall from [Alías et al. 2011] the concept of an Omori-Yau pair on a Riemannian manifold and discuss some implications of its existence.

Theorem 1. Let $f: M^{m} \rightarrow L^{\ell} \times{ }_{\rho} P^{n}$ be an isometric immersion, where $L^{\ell}$ carries an Omori-Yau pair for the Hessian and the functions $\rho$ and $|\operatorname{grad} \log \rho|$ are bounded. If $f$ is proper and $f(M) \subset \mathscr{C}_{r_{0}}$, then $\sup _{M}|H|=+\infty$ or

$$
\sup _{M} \rho|H| \geq \frac{m-\ell}{m} C_{b}\left(r_{0}\right),
$$

where $H$ is the mean curvature vector field of $f$.

In the proof, we see that the existence in $L^{\ell}$ of an Omori-Yau pair for the Hessian provides conditions, in a function-theoretic form, that guarantee the validity of the Omori-Yau maximum principle on $M^{m}$ in terms of the corresponding property of $L^{\ell}$ and the geometry of the immersion.

Definition 2. The pair of functions $(h, \gamma)$, for $h: \mathbb{R}_{+} \rightarrow \mathbb{R}_{+}$and $\gamma: M \rightarrow \mathbb{R}_{+}$, is an Omori-Yau pair for the Hessian in $M$ if

(a) $h(0)>0$ and $h^{\prime}(t) \geq 0$, for all $t \in \mathbb{R}_{+}$;

(b) $\limsup t h(\sqrt{t}) / h(t)<+\infty$;

$t \rightarrow+\infty$

(c) $\int_{0}^{+\infty} \frac{\mathrm{d} t}{\sqrt{h(t)}}=+\infty$;

(d) the function $\gamma$ is proper;

(e) $|\operatorname{grad} \gamma| \leq c \sqrt{\gamma}$ for some $c>0$ outside a compact subset of $M$; and

(f) Hess $\gamma \leq d \sqrt{\gamma h(\sqrt{\gamma})}$ for some $d>0$ outside a compact subset of $M$.

Similarly, the pair $(h, \gamma)$ is an Omori-Yau pair for the Laplacian in $M$ if it satisfies conditions (a)-(e) and

(f') $\Delta \gamma \leq d \sqrt{\gamma h(\sqrt{\gamma})}$ for some $d>0$ outside a compact subset of $M$. 
We say that the Omori-Yau maximum principle for the Hessian holds for $M$ if for any function $g \in C^{\infty}(M)$ bounded from above there exists a sequence of points $\left\{p_{k}\right\}_{k \in \mathbb{N}}$ in $M$ such that

(a) $\lim _{k \rightarrow \infty} g\left(p_{k}\right)=\sup _{M} g$,

(b) $\left|\operatorname{grad} g\left(p_{k}\right)\right| \leq 1 / k$,

(c) Hess $g\left(p_{k}\right)(X, X) \leq(1 / k) g_{M}(X, X)$ for all $X \in T_{p_{k}} M$.

Similarly, the Omori-Yau maximum principle for the Laplacian holds for $M$ if these properties are satisfied with (c) replaced by

$\left(\mathrm{c}^{\prime}\right) \Delta g\left(p_{k}\right) \leq 1 / k$

The following theorem of Pigola, Rigoli, and Setti gives sufficient conditions for an Omori-Yau maximum principle to hold for a Riemannian manifold.

Theorem 3 [Pigola et al. 2005]. Assume that a Riemannian manifold M carries an Omori-Yau pair for the Hessian (resp. Laplacian). Then the Omori-Yau maximum principle for the Hessian (resp. Laplacian) holds in $M$.

Example 4. Let $M^{m}$ be a complete but noncompact Riemannian manifold, and write $r(y)=\operatorname{dist}_{M}(y, o)$ for some reference point $o \in M^{m}$. Assume that the radial sectional curvature of $M^{m}$ satisfies $K^{\mathrm{rad}} \geq-h(r)$, where the smooth function $h$ satisfies (a)-(c) in Definition 2 and is even at the origin, that is, $h^{(2 k+1)}(0)=0$ for $k \in \mathbb{N}$. Then, as shown in [Pigola et al. 2005], the functions $\left(h, r^{2}\right)$ form an Omori-Yau pair for the Hessian. As for the function $h$, one can choose

$$
h(t)=t^{2} \prod_{j=1}^{N}\left(\log ^{(j)}(t)\right)^{2}, \quad t \gg 1,
$$

where $\log ^{(j)}$ stands for the $j$-th iterated logarithm.

To conclude this section, we observe that Theorem 1 is sharp. This is clear from (1) by taking as $P^{n}$ a space-form and as $M$ the hypersurface $L^{\ell} \times S_{P}\left(r_{0}\right)$ in $N^{n+\ell}$. In view of Example 4, it also follows that by taking $L^{\ell}=\mathbb{R}^{\ell}$ and constant $\rho$ we recover the result in [Alías et al. 2009].

\section{The proof}

We first introduce some additional notations, and then recall a few basic facts on warped product manifolds.

Let $\langle$,$\rangle denote the metrics in N^{n+\ell}, L^{\ell}$ and $M^{m}$, while (, ) stands for the metric in $P^{n}$. The corresponding norms are || and \|\| . In addition, let $\nabla$ and $\widetilde{\nabla}$ denote the Levi-Civita connections in $M^{m}$ and $N^{n+\ell}$, respectively, and $\nabla^{L}$ and $\nabla^{P}$ the ones in $L^{\ell}$ and $P^{n}$. 
We always denote vector fields in $T L$ by $T, S$ and in $T P$ by $X, Y$. Also, we identify vector fields in $T L$ and $T P$ with basic vector fields in $T N$ by taking $T(x, y)=T(x)$ and $X(x, y)=X(y)$.

For the Lie-brackets of basic vector fields, we have that $[T, S] \in T L$ and $[X, Y] \in$ $T P$ are basic and that $[X, T]=0$. Then we have

$$
\begin{aligned}
\widetilde{\nabla}_{S} T & =\nabla_{S}^{L} T, \\
\widetilde{\nabla}_{X} T & =\widetilde{\nabla}_{T} X=T(\varrho) X, \\
\widetilde{\nabla}_{X} Y & =\nabla_{X}^{P} Y-\langle X, Y\rangle \operatorname{grad}^{L} \varrho,
\end{aligned}
$$

where the vector fields $X, Y$ and $T$ are basic and $\varrho=\log \rho$.

Our proof follows the main steps in [Alías et al. 2011], where the geometric situation considered differs from ours in that $f(M)$ there is contained in a cylinder of the form

$$
\left\{(x, y) \in N^{n+\ell}: x \in B_{L}\left(r_{0}\right) \text { and } y \in P^{n}\right\} .
$$

In fact, a substantial part of the argument is to show that the Omori-Yau pair for the Hessian in $L^{\ell}$ induces an Omori-Yau pair for the Laplacian for a noncompact $M^{m}$ when $|H|$ is bounded. Thus the Omori-Yau maximum principle for the Laplacian holds in $M^{m}$, and the proof follows from an application of the latter.

Suppose that $M^{m}$ is noncompact, and let $(h, \Gamma)$ be an Omori-Yau pair for the Hessian in $L^{\ell}$. For $p \in M^{m}$, write $f(p)=(x(p), y(p))$. Set $\tilde{\Gamma}(x, y)=\Gamma(x)$ for $(x, y) \in N^{n+\ell}$ and

$$
\gamma(p)=\tilde{\Gamma}(f(p))=\Gamma(x(p)) .
$$

We show next that $(h, \gamma)$ is an Omori-Yau pair for the Laplacian in $M^{m}$. First we argue that the function $\gamma$ is proper. To see this, let $p_{k} \in M^{m}$ be a divergent sequence, that is, $p_{k} \rightarrow \infty$ in $M^{m}$ as $k \rightarrow+\infty$. Thus, $f\left(p_{k}\right) \rightarrow \infty$ in $N^{n+\ell}$ because $f$ is proper. Because $f(M)$ lies inside a cylinder, $x\left(p_{k}\right) \rightarrow \infty$ in $L^{\ell}$. Hence, $\gamma\left(p_{k}\right) \rightarrow+\infty$ as $k \rightarrow+\infty$ because $\Gamma$ is proper, and thus $\gamma$ is proper.

It remains to verify conditions (e) and $\left(f^{\prime}\right)$ in Definition 2. We have from $\tilde{\Gamma}(x, y)=\Gamma(x)$ that

$$
\left\langle\operatorname{grad}^{N} \tilde{\Gamma}(x, y), X\right\rangle=0 .
$$

Thus

$$
\operatorname{grad}^{N} \tilde{\Gamma}(x, y)=\operatorname{grad}^{L} \Gamma(x) .
$$

Since $\gamma=\tilde{\Gamma} \circ f$, we obtain

$$
\operatorname{grad}^{N} \tilde{\Gamma}(f(p))=\operatorname{grad}^{M} \gamma(p)+\operatorname{grad}^{N} \tilde{\Gamma}(f(p))^{\perp},
$$

where ()$^{\perp}$ denotes taking the normal component to $f$. Then

$$
\left|\operatorname{grad}^{M} \gamma(p)\right| \leq\left|\operatorname{grad}^{N} \tilde{\Gamma}(f(p))\right|=\left|\operatorname{grad}^{L} \Gamma(x(p))\right| \leq c \sqrt{\Gamma(x(p))}=c \sqrt{\gamma(p)}
$$

outside a compact subset of $M^{m}$, and thus (e) holds. 
We have that

$$
\widetilde{\nabla}_{T} \operatorname{grad}^{N} \tilde{\Gamma}=\nabla_{T}^{L} \operatorname{grad}^{L} \Gamma .
$$

Hence Hess $\tilde{\Gamma}(T, S)=\operatorname{Hess} \Gamma(T, S)$ and Hess $\tilde{\Gamma}(T, X)=0$. Also,

$$
\widetilde{\nabla}_{X} \operatorname{grad}^{N} \tilde{\Gamma}=\widetilde{\nabla}_{X} \operatorname{grad}^{L} \Gamma=\operatorname{grad}^{L} \Gamma(\varrho) X .
$$

Hence

$$
\text { Hess } \tilde{\Gamma}(X, Y)=\left\langle\operatorname{grad}^{L} \Gamma, \operatorname{grad}^{L} \varrho\right\rangle\langle X, Y\rangle .
$$

For a unit vector $e \in T_{p} M$, set $e=e^{L}+e^{P}$, where $e^{L} \in T_{x(p)} L$ and $e^{P} \in T_{y(p)} P$. Then

Hess $\tilde{\Gamma}(f(p))(e, e)=\operatorname{Hess} \Gamma(x(p))\left(e^{L}, e^{L}\right)+\left\langle\operatorname{grad}^{L} \Gamma(x(p)), \operatorname{grad}^{L} \varrho(x(p))\right\rangle\left|e^{P}\right|^{2}$. Also, an easy computation using (2) yields

$$
\text { Hess } \gamma(p)(e, e)=\text { Hess } \tilde{\Gamma}(f(p))(e, e)+\left\langle\operatorname{grad}^{L} \Gamma(x(p)), \alpha(p)(e, e)\right\rangle,
$$

where $\alpha$ denotes the second fundamental of $f$ with values in the normal bundle. Thus,

Hess $\gamma(p)(e, e)=\operatorname{Hess} \Gamma(x(p))\left(e^{L}, e^{L}\right)+\left\langle\operatorname{grad}^{L} \Gamma(x(p)),\left.\operatorname{grad}^{L} \varrho(x(p))|| e^{P}\right|^{2}\right.$

$$
+\left\langle\operatorname{grad}^{L} \Gamma(x(p)), \alpha(p)(e, e)\right\rangle .
$$

Since Hess $\Gamma \leq d \sqrt{\Gamma h(\sqrt{\Gamma})}$ for some positive constant $d$ outside a compact subset of $L^{\ell}$ and the immersion is proper, we have

$$
\text { Hess } \Gamma(x(p))\left(e^{L}, e^{L}\right) \leq d \sqrt{\gamma(p) h(\sqrt{\gamma(p)})}\left|e^{L}\right|^{2} \leq d \sqrt{\gamma(p) h(\sqrt{\gamma(p)})}
$$

outside a compact subset of $M^{m}$. From $\left|\operatorname{grad}^{L} \Gamma\right| \leq c \sqrt{\Gamma h(\sqrt{\Gamma})}$ for some $c$ outside a compact subset of $L^{\ell}$ and $\sup _{L}\left|\operatorname{grad}^{L} \varrho\right|<+\infty$, we have

$$
\left\langle\operatorname{grad}^{L} \Gamma(x(p)),\left.\operatorname{grad}^{L} \varrho(x(p))|| e^{P}\right|^{2} \leq c^{\prime} \sqrt{\gamma(p)}\right.
$$

for some positive constant $c^{\prime}$ outside a compact subset of $M^{m}$. Since $\gamma$ is proper and $h$ is unbounded, by (a) and (b) in Definition 2, we have

$$
\sqrt{\gamma} \leq \sqrt{\gamma h(\sqrt{\gamma})}
$$

outside a compact subset of $M^{m}$, because $\gamma \rightarrow+\infty$ as $p \rightarrow \infty$ and $\lim _{t \rightarrow+\infty} h(t)=$ $+\infty$. Thus we obtain

$$
\text { Hess } \gamma(e, e) \leq d_{1} \sqrt{\gamma h(\sqrt{\gamma})}+\left\langle\operatorname{grad}^{L} \Gamma(x), \alpha(e, e)\right\rangle
$$

for some constant $d_{1}>0$, outside a compact subset of $M^{m}$.

On the other hand, we may assume that

$$
|H| \leq c \sqrt{h(\sqrt{\gamma})}
$$


for some constant $c>0$, outside a compact subset of $M^{m}$. Otherwise, there exists a sequence $\left\{p_{k}\right\}_{k \in \mathbb{N}}$ in $M^{m}$ such that $p_{k} \rightarrow \infty$ as $k \rightarrow+\infty$ and

$$
\left|H\left(p_{k}\right)\right|>k \sqrt{h\left(\sqrt{\gamma\left(p_{k}\right)}\right)} \text {. }
$$

With $\gamma$ being proper and $h$ unbounded from (a) and (b) in Definition 2, we conclude that $\sup _{M}|H|=+\infty$, in which case we are done with the proof of the theorem.

We obtain from (3) using (4) that $\Delta \gamma \leq c_{1} \sqrt{\gamma h(\sqrt{\gamma})}$ for some constant $c_{1}>0$ outside a compact subset of $M^{m}$, and thus ( $\left.\mathrm{f}^{\prime}\right)$ has been proved.

Consider the distance function $r(y)=\operatorname{dist}_{P}(y, o)$ in $B_{P}\left(r_{0}\right)$ and define $\tilde{r} \in$ $C^{\infty}(N)$ by $\tilde{r}(x, y)=r(y)$. Then

$$
\left\langle\operatorname{grad}^{N} \tilde{r}(x, y), T\right\rangle=0 .
$$

Thus

$$
\rho^{2}(x) \operatorname{grad}^{N} \tilde{r}(x, y)=\operatorname{grad}^{P} r(y) .
$$

We obtain that

$$
\widetilde{\nabla}_{T} \operatorname{grad}^{N} \tilde{r}=\widetilde{\nabla}_{T}\left(\rho^{-2} \operatorname{grad}^{P} r\right)=-\rho^{-2} T(\varrho) \operatorname{grad}^{P} r .
$$

Therefore

$$
\text { Hess } \tilde{r}(T, S)=0
$$

and

$$
\text { Hess } \tilde{r}(T, X)=-\rho^{-2} T(\varrho)\left\langle\operatorname{grad}^{P} r, X\right\rangle=-T(\varrho)\left(\operatorname{grad}^{P} r, X\right) \text {. }
$$

Also,

$$
\widetilde{\nabla}_{X} \operatorname{grad}^{N} \tilde{r}=\widetilde{\nabla}_{X}\left(\rho^{-2} \operatorname{grad}^{P} r\right)=\rho^{-2}\left(\nabla_{X}^{P} \operatorname{grad}^{P} r-\left\langle X, \operatorname{grad}^{P} r\right\rangle \operatorname{grad}^{L} \varrho\right) .
$$

Hence

Hess $\tilde{r}(X, Y)=\rho^{-2}\left\langle\nabla_{X}^{P} \operatorname{grad}^{P} r, Y\right\rangle=\left(\nabla_{X}^{P} \operatorname{grad}^{P} r, Y\right)=$ Hess $r(X, Y)$.

For $e \in T M$, we have

$$
\text { Hess } \tilde{r}(e, e)=-2\left\langle\operatorname{grad}^{L} \varrho, e\right\rangle\left(\operatorname{grad}^{P} r, e^{P}\right)+\operatorname{Hess} r\left(e^{P}, e^{P}\right) .
$$

From the Hessian comparison theorem (see [Pigola et al. 2008, Chapter 2] for a modern approach) we obtain

$$
\text { Hess } r\left(e^{P}, e^{P}\right) \geq C_{b}(r)\left(\left\|e^{P}\right\|^{2}-\left(\operatorname{grad}^{P} r, e^{P}\right)^{2}\right) .
$$

Therefore,

(5) Hess $\tilde{r}(e, e) \geq-2\left\langle\operatorname{grad}^{L} \varrho, e\right\rangle\left(\operatorname{grad}^{P} r, e^{P}\right)+C_{b}(r)\left(\left\|e^{P}\right\|^{2}-\left(\operatorname{grad}^{P} r, e^{P}\right)^{2}\right)$.

We define $u \in C^{\infty}(M)$ by

$$
u(p)=r(y(p))
$$


Thus, $u=\tilde{r} \circ f$ and

$$
\operatorname{grad}^{N} \tilde{r}(f(p))=\operatorname{grad}^{M} u(p)+\operatorname{grad}^{N} \tilde{r}(f(p))^{\perp} .
$$

This gives

$$
\operatorname{Hess} u\left(e_{i}, e_{j}\right)=\operatorname{Hess} \tilde{r}\left(e_{i}, e_{j}\right)+\left\langle\operatorname{grad}^{N} \tilde{r}, \alpha\left(e_{i}, e_{j}\right)\right\rangle,
$$

where $e_{1}, \ldots, e_{m}$ is an orthonormal frame of $T M$. Thus

$$
\Delta u=\sum_{j=1}^{m} \operatorname{Hess} \tilde{r}\left(e_{j}, e_{j}\right)+m\left\langle\operatorname{grad}^{N} \tilde{r}, H\right\rangle .
$$

We have from $e_{j}=e_{j}^{L}+e_{j}^{P}$ that $1=\left\langle e_{j}, e_{j}\right\rangle=\rho^{2}\left\|e_{j}^{P}\right\|^{2}+\sum_{k=1}^{\ell}\left\langle e_{j}, T_{k}\right\rangle^{2}$, where $T_{1}, \ldots, T_{\ell}$ is an orthonormal frame for $T L$. Hence

$$
m=\rho^{2} \sum_{j=1}^{m}\left\|e_{j}^{P}\right\|^{2}+\sum_{k=1}^{\ell}\left|T_{k}^{\top}\right|^{2},
$$

where $T^{\top}$ is the tangent component of $T$. We obtain

$$
\sum_{j=1}^{m}\left\|e_{j}^{P}\right\|^{2} \geq(m-\ell) \rho^{-2}
$$

Since $\left(\operatorname{grad}^{P} r, e_{j}^{P}\right)=\left\langle\operatorname{grad}^{N} \tilde{r}, e_{j}^{P}\right\rangle=\left\langle\operatorname{grad}^{N} \tilde{r}, e_{j}\right\rangle=\left\langle\operatorname{grad}^{M} u, e_{j}\right\rangle$, we get from (5) that

$$
\text { Hess } \tilde{r}\left(e_{j}, e_{j}\right) \geq-2\left\langle\operatorname{grad}^{L} \varrho, e_{j}\right\rangle\left\langle\operatorname{grad}^{M} u, e_{j}\right\rangle+C_{b}(u)\left(\left\|e_{j}^{P}\right\|^{2}-\left\langle\operatorname{grad}^{M} u, e_{j}\right\rangle^{2}\right) \text {. }
$$

Taking the trace and using (8) gives

$$
\sum_{j=1}^{m} \operatorname{Hess} \tilde{r}\left(e_{j}, e_{j}\right) \geq-2\left\langle\operatorname{grad}^{L} \varrho, \operatorname{grad}^{M} u\right\rangle+C_{b}(u)\left((m-\ell) \rho^{-2}-\left|\operatorname{grad}^{M} u\right|^{2}\right) .
$$

Because $\left\langle\operatorname{grad}^{N} \tilde{r}, \operatorname{grad}^{N} \tilde{r}\right\rangle=\rho^{2}\left(\rho^{-2} \operatorname{grad}^{P} r, \rho^{-2} \operatorname{grad}^{P} r\right)=\rho^{-2}$, we have

$$
\left\langle\operatorname{grad}^{N} \tilde{r}, H\right\rangle \geq-\rho^{-1}|H| .
$$

Using (7), we conclude that

$$
\Delta u \geq-2\left\langle\operatorname{grad}^{L} \varrho, \operatorname{grad}^{M} u\right\rangle+C_{b}(u)\left((m-\ell) \rho^{-2}-\left|\operatorname{grad}^{M} u\right|^{2}\right)-m \rho^{-1}|H| .
$$

Thus

$$
\rho|H| \geq \frac{m-\ell}{m} C_{b}(u)-\frac{\rho^{2}}{m}\left(\Delta u+2\left|\operatorname{grad}^{L} \varrho\right|\left|\operatorname{grad}^{M} u\right|+C_{b}(u)\left|\operatorname{grad}^{M} u\right|^{2}\right) .
$$


If $M^{m}$ is compact, the proof follows easily by computing the inequality at a point of maximum of $u$. Thus, we may now assume that $M^{m}$ is noncompact and that (4) holds.

Since $f(M) \subset \mathscr{C}_{r_{0}}$, we have $u^{*}=\sup _{M} u \leq r_{0}<+\infty$. By the Omori-Yau maximum principle, there is a sequence $\left\{p_{k}\right\}_{k \in \mathbb{N}}$ in $M^{m}$ such that $u\left(p_{k}\right)>u^{*}-1 / k$, $\left|\operatorname{grad}^{M} u\left(p_{k}\right)\right|<1 / k$, and $\Delta u\left(p_{k}\right)<1 / k$. By assumption, we have $\sup _{L} \rho=K_{1}<$ $+\infty$ and $\sup _{L}\left|\operatorname{grad}^{L} \varrho\right|=K_{2}<+\infty$. Hence

$$
\sup _{M} \rho|H| \geq \rho\left(p_{k}\right)\left|H\left(p_{k}\right)\right| \geq \frac{m-\ell}{m} C_{b}\left(u\left(p_{k}\right)\right)-\frac{K_{1}^{2}}{m}\left(\frac{1+2 K_{2}}{k}+\frac{1}{k^{2}} C_{b}\left(u\left(p_{k}\right)\right)\right) .
$$

Letting $k \rightarrow+\infty$, we obtain

$$
\sup _{M} \rho|H| \geq \frac{m-\ell}{m} C_{b}\left(u^{*}\right) \geq \frac{m-\ell}{m} C_{b}\left(r_{0}\right),
$$

and this concludes the proof of the theorem.

\section{Acknowledgements}

This research is a result of the activity developed within the framework of the Programme in Support of Excellence Groups of the Región de Murcia, Spain, by Fundación Séneca, Regional Agency for Science and Technology (Regional Plan for Science and Technology 2007-2010). Alías was partially supported by MICINN project MTM2009-10418, MEC project PHB2010-0137-PC and Fundación Séneca project 04540/GERM/06, Spain. Dajczer was partially supported by CNPq and FAPERJ, Brazil.

\section{References}

[Alías et al. 2009] L. J. Alías, G. P. Bessa, and M. Dajczer, "The mean curvature of cylindrically bounded submanifolds", Math. Ann. 345:2 (2009), 367-376. MR 2010i:53104 Zbl 1200.53050

[Alías et al. 2011] L. J. Alías, G. P. Bessa, J. F. Montenegro, and P. Piccione, "Curvature estimates for submanifolds in warped products", Results Math. 60 (2011), 265-286.

[Greene and $\mathrm{Wu} 1979] \mathrm{R}$. E. Greene and H. Wu, Function theory on manifolds which possess a pole, Lecture Notes in Mathematics 699, Springer, Berlin, 1979. MR 81a:53002 Zbl 0414.53043

[Petersen 2006] P. Petersen, Riemannian geometry, 2nd ed., Graduate Texts in Mathematics 171, Springer, New York, 2006. MR 2007a:53001 Zbl 1220.53002

[Pigola et al. 2005] S. Pigola, M. Rigoli, and A. G. Setti, "Maximum principles on Riemannian manifolds and applications", Mem. Amer. Math. Soc. 174:822 (2005), x+99. MR 2006b:53048 Zbl 1075.58017

[Pigola et al. 2008] S. Pigola, M. Rigoli, and A. G. Setti, Vanishing and finiteness results in geometric analysis, Progress in Mathematics 266, Birkhäuser, Basel, 2008. MR 2009m:58001 Zbl 1150. 53001

Received December 30, 2010. Revised June 28, 2011. 
LUIS J. ALÍAS

DEPARTAMENTo DE MATEMÁticas

UNIVERSIDAD DE MURCIA

CAMPUS DE ESPINARDO

E-30100 ESPINARdo, MURCIA

SPAIN

ljalias@um.es

MARCOS DAJCZER

Instituto Nacional de Matemática Pura e Aplicada

Estrada Dona CASTORINA, 110

22460-320 RIO DE JANEIRO

BRAZIL

marcos@impa.br 


\title{
PACIFIC JOURNAL OF MATHEMATICS
}

\author{
http://pacificmath.org \\ Founded in 1951 by \\ E. F. Beckenbach (1906-1982) and F. Wolf (1904-1989)
}

\section{EDITORS}

V. S. Varadarajan (Managing Editor)

Department of Mathematics

University of California

Los Angeles, CA 90095-1555

pacific@math.ucla.edu

Vyjayanthi Chari

Department of Mathematics

University of California

Riverside, CA 92521-0135

chari@math.ucr.edu

\section{Robert Finn}

Department of Mathematics Stanford University

Stanford, CA 94305-2125

finn@math.stanford.edu

Kefeng Liu

Department of Mathematics

University of California

Los Angeles, CA 90095-1555

liu@math.ucla.edu
Darren Long

Department of Mathematics

University of California

Santa Barbara, CA 93106-3080

long@math.ucsb.edu

Jiang-Hua Lu

Department of Mathematics

The University of Hong Kong

Pokfulam Rd., Hong Kong jhlu@maths.hku.hk

Alexander Merkurjev

Department of Mathematics

University of California

Los Angeles, CA 90095-1555

merkurev@math.ucla.edu
Sorin Popa

Department of Mathematics University of California

Los Angeles, CA 90095-1555 popa@math.ucla.edu

Jie Qing

Department of Mathematics

University of California

Santa Cruz, CA 95064

qing@cats.ucsc.edu

Jonathan Rogawski

Department of Mathematics

University of California

Los Angeles, CA 90095-1555

jonr@math.ucla.edu

\section{PRODUCTION}

pacific@math.berkeley.edu

\section{SUPPORTING INSTITUTIONS}

ACADEMIA SINICA, TAIPEI

CALIFORNIA INST. OF TECHNOLOGY INST. DE MATEMÁTICA PURA E APLICADA KEIO UNIVERSITY

MATH. SCIENCES RESEARCH INSTITUTE NEW MEXICO STATE UNIV.

OREGON STATE UNIV.

\author{
STANFORD UNIVERSITY \\ UNIV. OF BRITISH COLUMBIA \\ UNIV. OF CALIFORNIA, BERKELEY \\ UNIV. OF CALIFORNIA, DAVIS \\ UNIV. OF CALIFORNIA, LOS ANGELES \\ UNIV. OF CALIFORNIA, RIVERSIDE \\ UNIV. OF CALIFORNIA, SAN DIEGO \\ UNIV. OF CALIF., SANTA BARBARA
}

\author{
UNIV. OF CALIF., SANTA CRUZ \\ UNIV. OF MONTANA \\ UNIV. OF OREGON \\ UNIV. OF SOUTHERN CALIFORNIA \\ UNIV. OF UTAH \\ UNIV. OF WASHINGTON \\ WASHINGTON STATE UNIVERSITY
}

These supporting institutions contribute to the cost of publication of this Journal, but they are not owners or publishers and have no responsibility for its contents or policies.

See inside back cover or pacificmath.org for submission instructions.

The subscription price for 2011 is US \$420/year for the electronic version, and \$485/year for print and electronic.

Subscriptions, requests for back issues from the last three years and changes of subscribers address should be sent to Pacific Journal of Mathematics, P.O. Box 4163, Berkeley, CA 94704-0163, U.S.A. Prior back issues are obtainable from Periodicals Service Company, 11 Main Street, Germantown, NY 12526-5635. The Pacific Journal of Mathematics is indexed by Mathematical Reviews, Zentralblatt MATH, PASCAL CNRS Index, Referativnyi Zhurnal, Current Mathematical Publications and the Science Citation Index.

The Pacific Journal of Mathematics (ISSN 0030-8730) at the University of California, c/o Department of Mathematics, 969 Evans Hall, Berkeley, CA 94720-3840, is published monthly except July and August. Periodical rate postage paid at Berkeley, CA 94704, and additional mailing offices. POSTMASTER: send address changes to Pacific Journal of Mathematics, P.O. Box 4163, Berkeley, CA 94704-0163.

PJM peer review and production are managed by EditFLOW ${ }^{\mathrm{TM}}$ from Mathematical Sciences Publishers.

PUBLISHED BY PACIFIC JOURNAL OF MATHEMATICS

at the University of California, Berkeley 94720-3840

A NON-PROFIT CORPORATION

Typeset in LATEX

Copyright $(2011$ by Pacific Journal of Mathematics 


\section{PACIFIC JOURNAL OF MATHEMATICS}

Volume $254 \quad$ No. $1 \quad$ November 2011

A mean curvature estimate for cylindrically bounded submanifolds

Luis J. AlíAS and MARCos DAJCZER

Weyl group multiple Dirichlet series of type $C$

JENNIFER BEINEKE, BENJAMIN BRUBAKER and SHARON FrECHETTE

Milnor open books of links of some rational surface singularities

MOHAN BHUPAL and BURAK OZBAGCI

Simple closed curves, word length, and nilpotent quotients of free groups

KHALID BOU-RABEE and ASAF HADARI

Strong submodules of almost projective modules

GÁBOR BRAUN and JAN TRLIFAJ

Interlacing log-concavity of the Boros-Moll polynomials

William Y. C. Chen, LARry X. W. WAng and ERnest X. W. Xia

Schwarzian norms and two-point distortion

Martin Chuaqui, Peter Duren, William Ma, Diego Mejía, David

MINDA and BRAD OSGOOD

The principle of stationary phase for the Fourier transform of $D$-modules

JIANGXUE FANG

Monotonicity and uniqueness of a 3D transonic shock solution in a conic nozzle with

variable end pressure

JUN LI, ZHOUPING XIN and HUICHENG YIN

Refined open noncommutative Donaldson-Thomas invariants for small crepant resolutions

KENTARO NAGAO

The Dirichlet problem for harmonic functions on compact sets

TONY L. PERKINS

Extension of an analytic disc and domains in $\mathbb{C}^{2}$ with noncompact automorphism group

\section{MINJU SONG}

Regularity of the first eigenvalue of the $p$-Laplacian and Yamabe invariant along geometric flows

ER-MIN WANG and YU ZHENG 